\title{
A first editorial
}

\author{
Robert J. Hall
}

Received: 14 June 2009 / Accepted: 17 June 2009 / Published online: 27 June 2009

(C) Springer Science+Business Media, LLC 2009

Greetings, ASE Community! It is my honor and pleasure this year to take over as Editor in Chief of the ASE Journal. In this, my first editorial, I would like to take a bit of space to thank my predecessor and to speak a bit about the challenges for the years ahead.

Professor Bashar Nuseibeh has served the ASE Community as Editor in Chief of this journal for ten-plus years. During his tenure, the ASE Journal has grown in scope to encompass all aspects of automation in software engineering, having started out more narrowly focused on artificial intelligence and knowledge-based techniques. This increase in scope has matched the scope increase of the ASE Conference series, and has been beneficial to the journal and community; not only do we get to learn about a much wider range of interesting automation techniques and theories, but our pool of excellent authors is larger as well.

In addition to nurturing the larger scope, Bashar has consistently applied the highest standards of quality in peer reviewing. Those of us who have managed to publish articles in the Journal well know how difficult it has been to get our work past Bashar's eagle eye and those of the reviewers he has overseen. This quality standard obviously benefits you, the readers, because you know that if articles appear here they are worth reading. They have long lasting archival value that will not disappear with the next release of a programming language, tool, or intellectual fad.

I hope I am not too presumptuous, then, in heartily thanking Bashar on behalf of myself, the ASE Journal, and indeed the entire ASE Community, for a job well done. Thanks, Bashar!

Opinions expressed here are solely those of the author and not necessarily those of AT\&T nor of Springer.

R.J. Hall ( $\varangle)$

AT\&T Labs Research, 180 Park Ave, Bldg 103, Florham Park, NJ 07932, USA

e-mail: Bob.ASEJ@gmail.com 
This all leaves me in the somewhat uncomfortable position of having a tough act to follow. Fortunately, Professor Nuseibeh has agreed to accept the designation Editor Emeritus, which allows me to ask him lots of questions and get his advice as I climb the learning curve.

Turning now to the future, the Journal finds itself in challenging times. The economic downturn, in affecting all businesses, challenges the publishing industry as well. Beyond that, scholarly communication, at least in software related fields, seems to be changing as well. Many authors seem to have less patience for journal publications, preferring to spend their time writing the next conference paper. Now, conferences are great for networking and discussing everyone's latest results, but in my opinion a conference's strict 5 to 10 page limits do not allow the full explanation of ideas together with details necessary for full understanding and replication. In short, conferences do not fill all the communication needs of a healthy scientific community.

The purpose and value of journal publication is to provide authors a chance to publish long-lived, archival results. It gives space to explain ideas well, to include details of experiments and case studies, and to discuss and relate the work fully into the tapestry of the surrounding research community. These are good reasons why journal publication has been the gold standard of scholarly output for so many years. I worry that a community that gives up on this mode of publication risks irrelevancy. In fifty years, will anyone bother sifting through tens of thousands of 5-10 page conference papers in a quixotic attempt to reconstruct the undisclosed details of some complex research project?

So consider redoubling your own efforts in contributing to the archival literature of our field. Urge your colleagues and students to contribute archival pieces here as well. If your work relates to software engineering automation, including the tools, processes, models, theories, frameworks, mathematics, and empirical studies underlying it, then the ASE Journal is the number one venue for your work; conversely, if the ASE Journal is to serve as a permanent record of the intellectual achievements in our community, it needs to have contributions from everyone doing significant work in our field. And, of course, if you have any questions about whether your work is in scope for the Journal, please send email and ask.

And now, lest you think the entire issue is one editorial, I would like to encourage you to explore the excellent articles in this issue.

- In "From UML/SPT models to schedulability analysis: approach and a prototype implementation using ATL", Gherbi and Khendek show how to transform UML models of real time systems into schedulability models for the systems using the ATLAS Transformation Language. Model transformation is an important topic in formal methods these days, as the modeling community has come to realize that a single model of a complex system just won't meet all needs.

- In "A semantic framework for metamodel-based languages", Gargantini, Riccobene, and Scandurra provide a formal semantic framework supporting the definition of metamodel-based languages and apply it to the OMG metamodeling framework. Another critical topic in modeling these days is pinning down the precise meaning of all those models, whether sketched on whiteboards, drawn in an editor, or produced via transformation from other models. 
- In "Automated software license analysis", Tuunanen, Koskinen, and Karkkainen take on the complex issue of how to reuse software legally, in accord with the diverse license terms that people put into such components. Their ASLA tool is capable of identifying licenses in source files and, using pre-stored knowledge of interactions, notifying the reuser of potential conflicts.

In summary, even as you enjoy the archival contributions in this issue, please keep working on your own ASEJ manuscript, and enjoin your colleagues to work on theirs. And please bear with me as I attempt to follow a class act. 